\title{
Bone marrow-derived, alternatively activated macrophages enhance solid tumor growth and lung metastasis of mammary carcinoma cells in a Balb/C mouse orthotopic model
}

Han Jin Cho', Jae In Jung ${ }^{1}$, Do Young Lim', Gyoo Taik Kwon'1, Song Her², Jong Hoon Park ${ }^{3}$ and Jung Han Yoon Park ${ }^{1 *}$

\begin{abstract}
Introduction: Tumor-associated macrophages, which are derived from the infiltration of circulating bone marrowderived monocytes, consist primarily of a polarized M2 macrophage (M2-M $\varphi$ ) population and are associated with poor prognosis in various cancers. In the present study, we attempted to assess whether M2-Mps derived from bone marrow stimulate the promotion and progression of mammary tumors.

Methods: 4T1 murine mammary carcinoma cells were injected either alone or coupled with M2-Mps into the mammary fat pads of syngeneic female Balb/C mice. M2-Mps were prepared by treating monocytes isolated from female Balb/C mouse bone marrow with IL-4. Tumor cell growth was determined using an in vivo imaging system and the expression of cell proliferation-related, angiogenesis-related, and lymphangiogenesis-related proteins in tumor tissues was immunohistochemically analyzed. To evaluate the effects of the crosstalk between 4T1 cells and M2-Mps on the secretion and mRNA expression of cytokines and the migration of monocytes, 4T1 cells and M2-

Mps were co-cultured and cytokine antibody array, real-time RT-PCR, and trans-well migration assays were conducted.

Results: The co-injection of M2-Mps into the mammary fat pads of mice increased solid tumor growth and lung metastasis of $4 \mathrm{~T} 1$ cells as well as the infiltration of $\mathrm{CD} 45^{+}$leukocytes into tumor tissues. The proportions of Ki- $67^{+}$ proliferating cells and the expression of hypoxia inducible factor-1 $\alpha$, vascular endothelial cell growth factor $A$, CD31, vascular endothelial cell growth factor C, and lymphatic vessel endothelial receptor-1 were increased significantly in the tumor tissues of mice co-injected with $4 \mathrm{~T} 1$ cells and M2-Mps. The in vitro results revealed that the proliferation of $4 \mathrm{~T} 1$ cells, the migration of monocytes, and the secretion of granulocyte colony-stimulating factor, IFN $\gamma$, IL-1 $\alpha$, IL-2, IL-16, IFN $\gamma$-induced protein-10, keratinocyte-derived chemokine, macrophage colonystimulating factor, monocyte chemotactic protein-1, macrophage inflammatory protein-1 $\alpha$, and RANTES were increased when $4 \mathrm{~T} 1$ cells were co-cultured with M2-M $\mathrm{MS}$, as compared with when the 4T1 cells were cultured alone.
\end{abstract}

Conclusion: The crosstalk between 4T1 cells and M2-Mps increased the production of cytokines, which may have induced immune cell infiltration into tumor tissues, tumor cell proliferation, angiogenesis, and lymph angiogenesis, thereby increasing solid tumor growth and lung metastasis.

\footnotetext{
* Correspondence: jyoon@hallym.ac.kr

1 Department of Food Science and Nutrition, Hallym University, Chuncheon

200-702, Korea

Full list of author information is available at the end of the article
} 


\section{Introduction}

Macrophages, which are derived from bone marrow progenitors, are recruited into tissues to replace resident populations or react to a variety of inflammatory and immune stimuli. The differentiated phenotype of recruited macrophages reflects signals from the microenvironment in which they dwell. These macrophages are broadly divided into two main classes; classically activated macrophages, or alternatively activated macrophages (M2-M $\varphi s)$. Classically activated macrophages have immunostimulatory, T-helper type 1-orienting properties and have an IL- ${ }^{\text {high }}$, IL- $6^{\text {high }}$, IL- $12^{\text {high }}$, IL$23^{\text {high }}$, TNF ${ }^{\text {high }}$, and IL-10 ${ }^{\text {low }}$ profile. By way of contrast, M2-M $\varphi$ s have an IL-1 ${ }^{\text {low }}, \mathrm{IL}_{-} 6^{\text {low }}, \mathrm{IL}-12^{\text {low }}, \mathrm{TNF}^{\text {low }}$, and IL-10 ${ }^{\text {high }}$ profile, poor antigen-presenting capacity, and are reported to suppress $\mathrm{T}$-helper type 1 adaptive immunity (reviewed in [1]).

Solid tumors consist of a number of cells, including malignant cells, fibroblasts, endothelial cells, and immune cells including macrophages. As cancer cells generate chemotactic factors for monocytes, macrophage accumulations were frequently observed in a variety of cancers, including breast cancer [2-4]. Tumor microenvironments produce a variety of factors, which lead to promotion of the differentiation and polarization of infiltrated monocytes into M2-M $\varphi s$ [5]. The macrophage within the tumor, which is referred to as tumor-associated macrophage (TAM), exhibits several pro-tumoral functions, including the promotion of angiogenesis, the suppression of adaptive immunity, and the expression of growth factors and matrix proteases (reviewed in [1]). Recent evidence has shown that TAM is associated with poor prognosis in cancers, including breast cancer, lung cancer, and pancreatic cancer $[2,4,6]$. In a mouse model of mammary tumors initiated by the expression of the polyoma virus middle $\mathrm{T}$ oncoprotein, the null mutation in the colony-stimulating factor-1 gene CSF-1 to deplete macrophages has been demonstrated to reduce the progression of preinvasive lesions to malignant lesions and attenuate the formation of lung metastasis [7]. Using a 4T1 orthotopic Balb/C mammary cancer model in which 4T1 mammary carcinoma cells were injected into the mammary fat pads of syngeneic Balb/C mice, Luo and colleagues have shown that a legumain-based DNA vaccine targeting TAM suppressed tumor angiogenesis and metastasis [8].

In this study, we evaluated the effect of M2-Mps on the promotion of mammary cancer using the 4T1orthotopic tumor model in which 4T1 mammary carcinoma cells were injected in conjunction with M2-M $\varphi$ s. The 4T1 cell line was derived from a naturally occurring mammary tumor in a Balb/C mouse [9] and thus the inoculated $4 \mathrm{~T} 1$ cells grow into solid tumors that spontaneously metastasize to the lung, whereas the original tumor continues to grow in the mammary fat pads. The $4 \mathrm{~T} 1$ model is thus a physiological mouse model that closely mimics breast cancer in humans $[9,10]$. To prepare M2-M $\varphi$ s, we isolated monocytes from the bone marrow of female Balb/C mice and treated them with IL-4. The M2-M $\varphi$ s and 4T1 cells are syngeneic in Balb/C mice, and - as opposed to immunedeficient nude mice, which are extensively used as in vivo tumor models - BALB/c mice are immune competent. This animal model is therefore useful in the study of tumor growth and progression, in which the involvement of the inflammatory/immune system is a critical consideration.

We used an in vivo imaging system to estimate the accumulation of $4 \mathrm{~T} 1$ tumor cells in the solid tumor. To quantify tumor cells, we engineered 4T1-luc cells that stably and constitutively expressed a firefly luciferase enzyme. The bioluminescence signal is derived from viable 4T1-luc cells only, not from other cell types present in a tumor such as fibroblasts, endothelial cells, and immune cells. Additionally, the in vivo imaging system is useful for repeatedly estimating the growth of a solid tumor over the course of an experiment, with each animal serving as its own reference. We demonstrated that the co-injection of bone marrow-derived M2-M with $4 \mathrm{~T} 1$ cells into mammary fat pads effectively stimulated solid tumor growth and lung metastasis.

\section{Materials and methods Antibodies}

The following antibodies were purchased from the suppliers as indicated: antibodies against Ki-67, hypoxiainducible factor-1 alpha (HIF-1 $\alpha$ ), and lymphatic vessel endothelial hyaluronan receptor-1 from Abcam (Cambridge, UK); antibodies against cyclin-dependent kinase (CDK)2, CDK4, vascular endothelial cell growth factor (VEGF)-A, VEGF-C, and CD31 from Santa Cruz Biotechnology (Santa Cruz, CA, USA); anti-CD45 antibody from R\&D Systems (Minneapolis, MN, USA); antibodies against rabbit-IgG-Alexa488, rabbit-IgG-Alexa597, and goat-IgG-Alexa488 from Invitrogen (Carlsbad, CA, USA); and anti-goat-IgG-Cy3 antibody from Rockland (Gibertsville, PA, USA). The primary and secondary antibodies were used at a dilution of 1:200 and 1:1,000, respectively.

\section{Cell culture}

The $4 \mathrm{~T} 1$ cell line was purchased from the American Type Culture Collection (Manassas, VA, USA). To establish a $4 \mathrm{~T} 1$ cell line that stably expresses firefly luciferase (4T1-luc), the cells were infected with a lentiviral vector harboring a gene encoding for firefly luciferase 
[11]. The 4T1 and 4T1-luc cell lines were maintained in DMEM containing 10\% FBS. The L929 murine fibrosarcoma cell line was purchased from the Korean Cell Line Bank (Seoul, Korea) and maintained in RPMI 1640 containing 10\% heat-inactivated FBS. All cultures contained $100 \mathrm{kU} / \mathrm{l}$ penicillin and $100 \mathrm{mg} / \mathrm{l}$ streptomycin.

\section{Preparation of $\mathrm{M} 2-\mathrm{M} \varphi \mathrm{s}$ derived from mouse bone marrow} Bone marrow cells were collected from the femoral shafts of female Balb/C mice. Monocytes were isolated from the bone marrow cell suspensions using the EasySep mouse monocyte negative-selection enrichment mixture (StemCell Technologies, Vancouver, Canada) in accordance with the manufacturer's protocols. The isolated monocytes were cultured and differentiated for 7 days in DMEM containing 10\% heat-inactivated FBS and $10 \%$ L929 cell-conditioned medium [12]. The cell monolayers were then treated overnight with IL-4 to induce differentiation into the M2 phenotype. To determine whether the macrophages evidence the characteristics of M2-M $\varphi s$, the expression of macrophage mannose receptors was routinely estimated via RT-PCR [13].

\section{Animals and in vivo imaging system}

Female Balb/C mice (3 weeks of age) were purchased from Orient Bio Inc. (Gapyung, Korea) and were acclimatized to laboratory conditions at the animal research facility of Hallym University, Chuncheon, Korea. All animal experimental protocols were approved by the Animal Care and Use Committee of Hallym University (Hallym2010-14). Balb/C mice were fed on an AIN-76A diet (Research Diets, New Brunswick, NJ, USA) and water ad libitum. After acclimatization, 4T1-luc cells $\left(5 \times 10^{4}\right.$ cells) and/or M2-M $\varphi$ s $\left(1 \times 10^{4}\right.$ cells $)$ were suspended in $0.1 \mathrm{ml}$ Matrigel (BD Biosciences, San Jose, CA, USA) and injected into the inguinal mammary fat pads of female Balb/C mice ( 4 weeks of age, eight mice/group).

For the bioluminescence imaging, mice received an intraperitoneal injection of $150 \mu \mathrm{l} \mathrm{D}$-luciferin $(30 \mathrm{mg} /$ $\mathrm{ml}$ ). Fifteen minutes after the injection of D-luciferin, the mice were anesthetized with isoflurane/oxygen and placed on the imaging stage. The bioluminescence signals were monitored using an IVIS-200 (Xenogen Corp., Alameda, CA, USA). The data were analyzed using the total photon flux emission (photons/second) in the regions of interest.

Three weeks after 4T1 cell injection, mice were anesthetized and the tumors were removed and weighed. The lungs were fixed in Bouin's solution, and the metastases were quantified.

To determine whether immune cells in the tumor tissues are derived from injected M2-M $\mathrm{Ms}$ or infiltrated circulating leukocytes, a separate animal experiment was conducted. For this experiment, M2-M $\varphi$ s were labeled with the green fluorescence-tagged lipophilic tracer 3,3'dioctadecyloxacarbocyanine perchlorate/ $\mathrm{DiOC}_{18}(\mathrm{DiO}$ Invitrogen) immediately prior to the injection. To accomplish this, the M2-M $\varphi$ s were exposed to $50 \mu \mathrm{mol} / \mathrm{l} \mathrm{DiO}$ for 2 hours and were washed with PBS. The animals (five mice/group) were sacrificed 2 weeks after the injection.

\section{Immunohistochemical and immunofluorescence staining}

For immunohistochemical staining, tumors were fixed in $4 \%$ paraformaldehyde and embedded in paraffin wax. Sections of $5 \mu \mathrm{m}$ were prepared from the paraffinembedded tumor blocks and hydrated through xylene and graded alcohol. Antigen retrieval was performed by microwaving the slides in $10 \mathrm{mmol} / \mathrm{l}$ sodium citrate buffer ( $\mathrm{pH}$ 6.0). Endogenous peroxidases were quenched with $3 \%$ hydrogen peroxide for 5 minutes. Sections were incubated with their relevant antibodies, and were then developed using an LSAB kit (Dako, Carpinteria, CA, USA) in accordance with the manufacturer's instructions.

For immunofluorescence staining, tumors were immersed in optimal cutting temperature compound and frozen rapidly in liquid nitrogen. Then, $5 \mu \mathrm{m} \mathrm{sec-}$ tions were cut with a cryostat microtome. Sections were fixed with $4 \%$ paraformaldehyde, permeabilized with icecold methanol, and incubated with their relevant antibodies. Nuclei were counterstained with 4,6-diamidino-2phenylindole.

\section{Co-culture of $4 \mathrm{~T} 1$ cells and $\mathrm{M} 2-\mathrm{M} \varphi s$}

To investigate the cytokine production, $4 \mathrm{~T} 1$ cells $(1 \times$ $10^{5}$ cells/well) were plated in 12 -well plates with or without M2-Mps $\left(2 \times 10^{4}\right.$ cells/well $)$. After 24 hours, cells were serum starved for 24 hours with DMEM. After serum starvation, cells were incubated in fresh DMEM for a further 24 hours, and the 24-hour-conditioned media were collected. Four independent experiments were conducted and the conditioned media were pooled. The relative levels of cytokines in the pooled conditioned media were measured via a mouse cytokine array panel A kit (ARY006) in accordance with the manufacturer's instructions (R\&D Systems). Densitometric analysis was conducted using the Bioprofile BioID application (Vilber Lourmat, Marne-la-Vallée, France). Expression levels were normalized to the levels of the positive control spots (contained within the membrane), and the positive control levels were set at $100 \%$.

To determine the expression of cytokine mRNAs in a co-culture model, 4T1 cells or M2-M $\varphi$ s were plated on cell culture coverslips $(24 \mathrm{~mm} \times 30 \mathrm{~mm})$ in eight-well plates (Nunc Inc., Rochester, NY, USA). After 24 hours, two coverslips (covered with the monolayers of 4T1 cells or M2-M $\varphi s$ ) were placed in the same wells of fourwell plates $(4 \mathrm{~T} 1+4 \mathrm{~T} 1 ; 4 \mathrm{~T} 1+\mathrm{M} 2-\mathrm{M} \varphi s$; or $\mathrm{M} 2-\mathrm{M} \varphi \mathrm{s}+$ M2-M $\varphi s$ ) and incubated in fresh DMEM for 24 hours. 
The total RNAs of each cell type were isolated using an RNeasy Plus Mini Kit (Qiagen, Valencia, CA, USA), and the real-time RT-PCR was conducted as previously described [14]. The sequences for PCR primer sets are listed in Table 1. The control levels (4T1 cells alone or M2-M $\varphi$ s cells alone) were set at 1 .

\section{Trans-well migration assays of bone marrow-derived monocytes}

Monocytes were isolated as described above and plated onto the type IV collagen-coated filter ( $3 \mu \mathrm{m}$ pore size) in 6.5 - $\mathrm{mm}$ trans-well inserts at $1 \times 10^{5}$ cells. The lower chamber of the well was filled with or without media conditioned by $4 \mathrm{~T} 1$ cells and/or M2-M $\varphi$ s. Monocytes were incubated for 1 hour. Migrated cells were stained with 4',6-diamidino-2-phenylindole and were quantified by counting the 4,6-diamidino-2-phenylindole-stained cells.

\section{Statistical analysis}

The results are expressed as the mean \pm standard error of the mean. Differences between groups were assessed via the Student's $t$ test and Duncan's multiple-range test, utilizing SAS statistical software, version 8.12 (SAS Institute, Cary, NC, USA).

\section{Results}

M2-Mps stimulate the solid tumor growth of 4T1 mammary cancer cells in Balb/C mice

To determine whether M2-M $\varphi$ s stimulate the growth of mammary cancer cells in vivo, we injected 4T1-luc cells

Table 1 Primer sequences used for real-time RT-PCR

\begin{tabular}{|c|c|c|}
\hline mRNA & Primer sequences & Reference \\
\hline \multirow[t]{2}{*}{ M-CSF } & Forward: 5'-TTGGCTTGGGATGATTCTCAG-3' & {$[28]$} \\
\hline & Backward: 5'-GCCCTGGGTCTGTCAGTCTC-3' & \\
\hline \multirow[t]{2}{*}{ IP-10 } & Forward: 5'-CCCACGTGTTGAGATCATTG-3' & [29] \\
\hline & Backward: 5'-GCTCTCTGCTGTCCATCCAT-3' & \\
\hline \multirow[t]{2}{*}{ RANTES } & Forward: 5'-CССТCACCATCATCCTCACT-3 ' & {$[30]$} \\
\hline & Backward: 5'-CCACTTCTTCTCTGGGTTGG-3' & \\
\hline \multirow[t]{2}{*}{ MCP-1 } & Forward: 5'-CAGCCAGATGCAGTTAACGC-3' & \\
\hline & Backward: 5'-CCTCTCTCTTGAGCTTGGTG-3' & \\
\hline \multirow[t]{2}{*}{ G-CSF } & Forward: 5'-CATGGCTCAACTTTCTGCCCA-3' & {$[31]$} \\
\hline & Backward: 5'-TAGGTGGCACACAACTGCTC-3' & \\
\hline \multirow[t]{2}{*}{$\mid L-1 \alpha$} & Forward: 5'-AAGACAAGCCTGTGTTGCTGAAGG-3' & {$[32]$} \\
\hline & Backward: 5'-CCAGAAGAAAATGAGGTCGGTC-3' & \\
\hline \multirow[t]{2}{*}{ KC } & Forward: 5'-CAAGAACATCCAGAGCTTGAAGGT-3' & {$[33]$} \\
\hline & Backward: 5'-GTGGCTATGACTTCGGTTTGG-3' & \\
\hline \multirow[t]{2}{*}{ MIP-1 $\alpha$} & Forward: 5'-ACCATGACACTCTGCAACCA-3' & [34] \\
\hline & Backward: 5'-CCCAGGTCTCTITGGAGTCA-3' & \\
\hline
\end{tabular}

G-CSF, granulocyte colony-stimulating factor; HIF-1 $\alpha$, hypoxia-inducible factor$1 \alpha$; IP-10, IFN $\gamma$-induced protein-10; KC, keratinocyte chemoattractant; M-CSF, macrophage colony-stimulating factor; $\mathrm{MCP}-1$, monocyte chemotactic protein1; MIP-1 $\alpha$, macrophage inflammatory protein-1 alpha; RANTES, regulated upon activation, normal T-cell expressed and secreted. into the mammary fat pads of Balb/C either alone or coupled with M2-M $\varphi$ s. Prior to the injection, the levels of macrophage mannose receptor transcripts were estimated via RT-PCR in order to determine whether the IL-4-treated cells exhibit an M2 phenotype. IL-4 treatment effectively induced the mRNA expression of the macrophage mannose receptor (data not shown). At the indicated time point after the tumor cell inoculation, all mice were scanned with the IVIS-200 system (Xenogen Corp.) and each bioluminescent signal was calculated. At 2 days after the inoculation, there was no significant difference in the bioluminescent signals between the two groups. Co-injection with M2-M $\varphi$ s significantly increased bioluminescent signals at 9 days after the inoculation and the increase in bioluminescent signals was more pronounced at 16 days (Figure 1A,B). These results clearly demonstrate that the accumulation of $4 \mathrm{~T} 1$ cancer cells in the mammary fat pads was increased significantly when the $4 \mathrm{~T} 1$ cells were co-injected with M2-M $\varphi s$ as compared with when $4 \mathrm{~T} 1$ cells were injected alone. In a different experiment (10 mice/group) we measured tumor volumes and weights, which were noted to be increased when $4 \mathrm{~T} 1$ cells were coinjected with M2-M $\varphi$ s (data not shown).

As the tumor growth was increased in mice coinjected with $4 \mathrm{~T} 1$ cells and M2-M $\mathrm{Ms}$, we subsequently evaluated the effects of $\mathrm{M} 2-\mathrm{M} \varphi \mathrm{s}$ on the expression levels of proteins involved in cell proliferation via immunohistochemistry. As compared with mice injected with 4T1 cells alone, the proportions of Ki-67-positive cells (Ki-67 is a nucleoprotein expressed only in cycling cells) and the expression of CDK2 and CDK4 were increased significantly in mice co-injected with M2-M $\varphi$ s (Figure 1C). To determine whether M2-M $\varphi$ s stimulate $4 \mathrm{~T} 1$ cell proliferation, an in vitro study was conducted by culturing 4T1-luc cells alone or with M2-M $\varphi$ s. A positive correlation was noted between 4T1-luc cell numbers and bioluminescent signals $\left(R^{2}=0.9988\right)$. Bioluminescent signals were significantly increased when the 4T1-luc cells were co-cultured for 4 days with M2-Mps compared with when 4T1-luc cells were cultured alone, indicating that the crosstalk between the two cell types induces the proliferation of 4T1 cells (Figure 1D).

\section{$\mathrm{M} 2-\mathrm{M} \varphi \mathrm{s}$ induce an increase in lung metastasis of 4T1 mammary cancer cells in Balb/C mice}

To assess the effect of M2-M $\varphi$ s on the lung metastasis of mammary cancer cells, 4T1-cell-bearing mice were sacrificed at 3 weeks after the inoculation, the lungs were fixed in Bouin's solution, and the metastases were quantified. At this time, tumor nodules were detected in all animals injected with $4 \mathrm{~T} 1$ cells. The number and volume of lung tumor nodules per mouse were increased significantly in animals co-injected with M2$\mathrm{M} \varphi s$ (Figure 2A to 2C). 


\section{A}

Days after injection

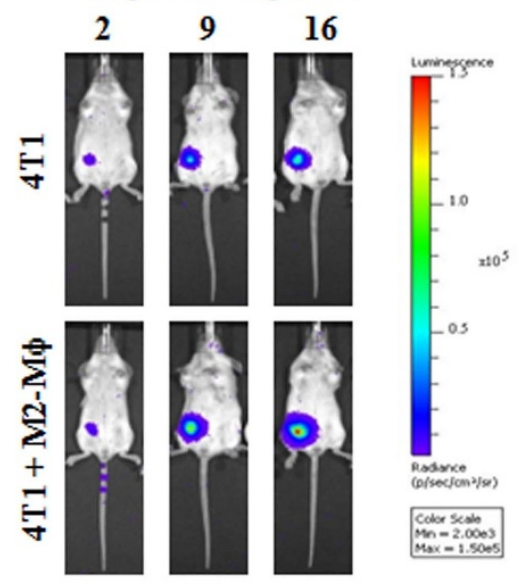

B

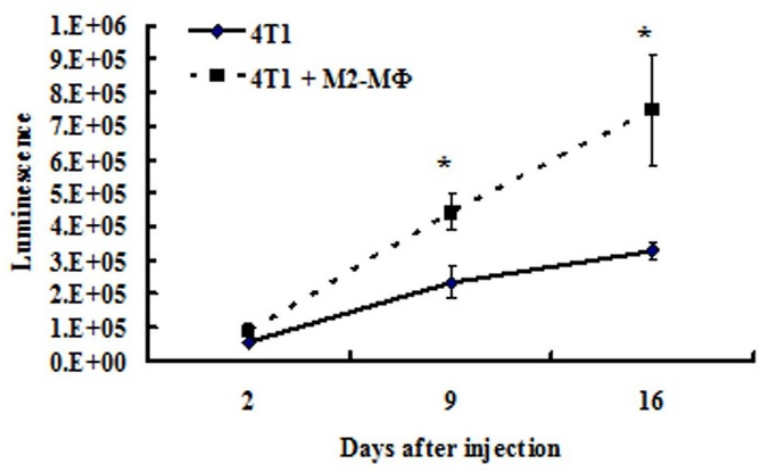

C

IHC:

Ki-67

CDK2

CDK4
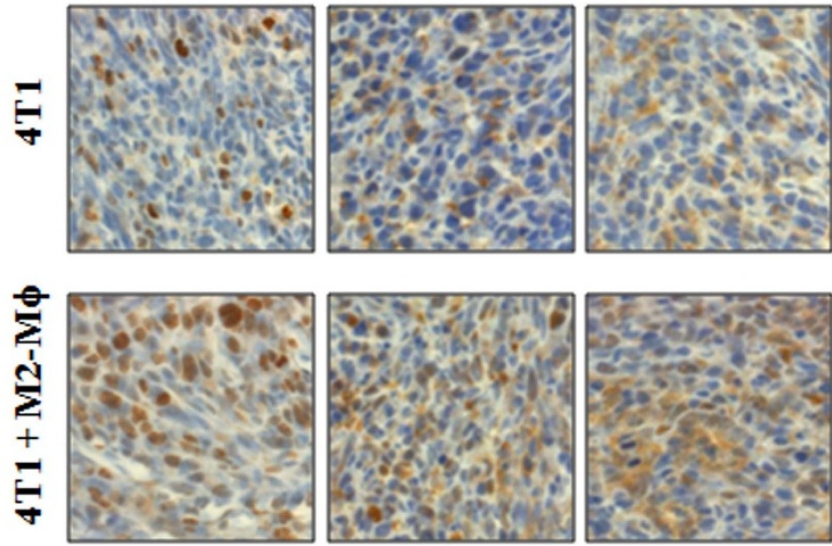

D
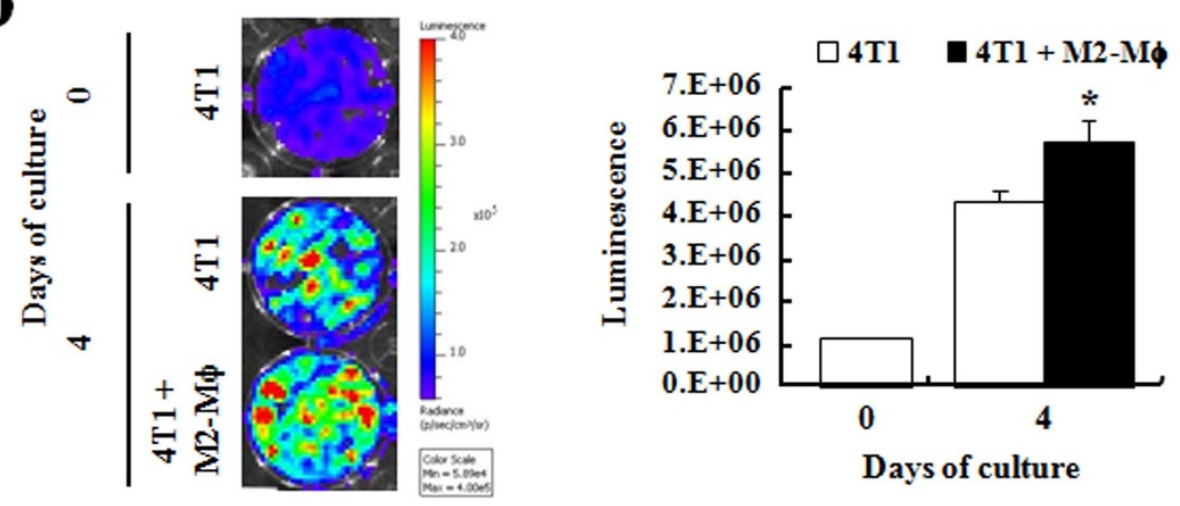

Figure 1 M2-polarized macrophages enhance tumor growth and increase cell proliferation indices. 4T1-luc cells and/or M2-polarized macrophages (M2-Mps) were injected into the mammary fat pads of female Balb/C mice. Bioluminescent signals were monitored on the days indicated. (A) Representative images of luciferase signals. (B) Quantitative analysis of luminescence. Each bar represents the mean \pm standard error of the mean (SEM) $(n=8)$. (C) Immunohistochemical $(I H C)$ staining for Ki-67, cyclin-dependent kinase (CDK)2, and CDK4 in tumor sections was based on 3,3'-diaminobenzidine staining, as described in the Materials and methods section. (D) 4T1-luc cells and/or M2-M $\varphi$ s were cultured in 24-well plates. Bioluminescent signals were monitored at the indicated day. Each bar represents the mean \pm SEM from three independent experiments performed in duplicate. *Significantly different from the 4T1 group, $P<0.05$. 


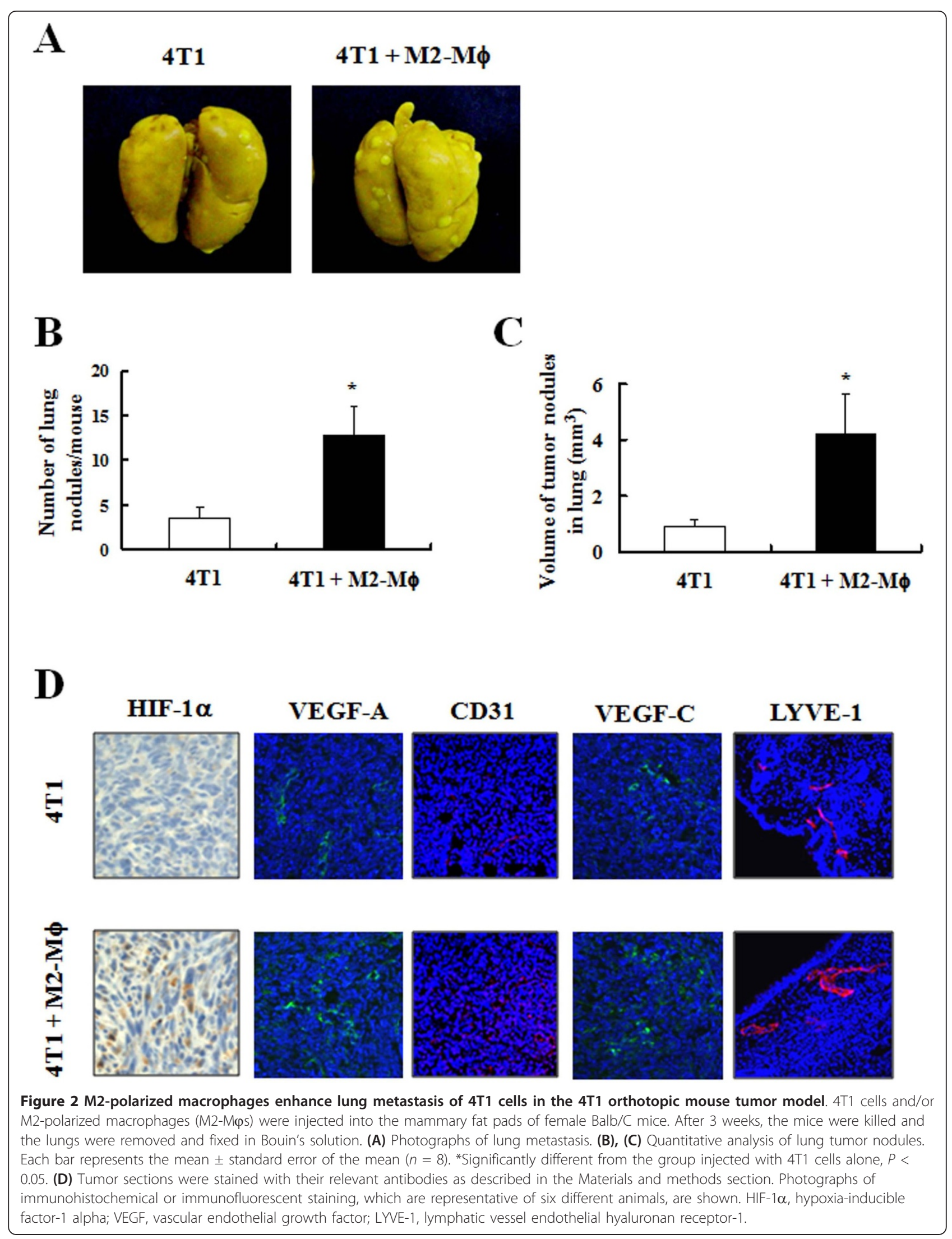


HIF- $1 \alpha$ activates the transcription of multiple genes including VEGF-A, a key factor in tumor angiogenesis. The accumulation of HIF- $1 \alpha$ in the nuclei of tumor cells was increased in mice co-injected with 4T1 cells and M2-M $\varphi$ s (Figure 2D). Co-inoculation of M2-M increased the expression of VEGF-A as well as the proportions of CD31-positive cells (CD31 is a glycoprotein expressed on endothelial cells and in platelets) (Figure 2D). Additionally, the co-inoculation of M2-M increased the expression of the lymphangiogenic growth factor VEGF-C and lymphatic vessel endothelial hyaluronan receptor-1-positive vessels.

\section{Crosstalk between 4T1 cells and M2-M $\varphi$ s increases the infiltration of monocytes into tumor tissues in Balb/C mice}

In an effort to determine whether the co-injection of $\mathrm{M} 2-\mathrm{M} \varphi s$ induces the infiltration of circulating monocytes into tumor tissues, M2-M $\varphi$ s were labeled with the green fluorescence-tagged, lipophilic tracer $\mathrm{DiO}$ immediately before being injected. At 2 weeks after the tumor cell injection, the injected M2-M $\varphi$ s (green color) were observed only in the central area of the tumor. Tumor sections were stained with a red fluorescence-tagged CD45 antibody. CD $45^{+}$cells (red color) were distributed diffusely throughout the tumor area and increased in mice co-injected with $4 \mathrm{~T} 1$ cells and M2-M $\mathrm{Ms}$ (Figure 3A). Additionally, in vitro trans-well migration assay results revealed that the migration of bone marrowderived monocytes was tremendously elevated when medium conditioned by $4 \mathrm{~T} 1 / \mathrm{M} 2-\mathrm{M} \varphi$ co-cultures was used as a chemoattractant, as compared with medium conditioned by 4T1 cells or M2-M 9 s alone (Figure 3B).

\section{Crosstalk between 4T1 cells and M2-M $\varphi$ s stimulates cytokine production}

The results of the mouse cytokine antibody array analyses indicated that the levels of granulocyte colony-stimulating factor (G-CSF), IFN $\gamma$, IL-1 $\alpha$, IL-2, IL-16, IFN $\gamma$ induced protein-10 (IP-10), keratinocyte-derived chemokine $(\mathrm{KC})$, macrophage colony-stimulating factor (MCSF), monocyte chemotactic protein-1 (MCP-1), macrophage inflammatory protein-1 $\alpha$ (MIP-1 $\alpha)$, and regulated upon activation, normal $\mathrm{T}$-cell expressed and secreted (RANTES) were increased in the media conditioned by the co-culture of 4T1 cells and M2-M $\varphi$ s (Figure 4A). The results of real-time RT-PCR demonstrated that the expression of M-CSF, IP-10, and RANTES mRNAs increased in 4T1 cells when 4T1 cells were co-cultured with $\mathrm{M} 2-\mathrm{M} \varphi$ s as compared with when 4T1 cells were cultured alone. In M2-M $\varphi$ s, the mRNA expression of GCSF, IL- $1 \alpha, \mathrm{KC}$, and MIP-1 $\alpha$ were increased when M2M $\varphi s$ were co-cultured with 4T1 cells as compared with when M2-M $\varphi$ s cells were cultured alone. The expression of MCP-1 mRNA increased in both 4T1 cells and M2M $\varphi s$ upon co-culture (Figure 4B). These results demonstrate that interactions between 4T1 cells and M2-M 9 s increase the production of a variety of chemoattractants, resulting in increases in tumor cell proliferation and monocyte migration.

\section{Discussion}

The tumor microenvironment consists of several components, including malignant cells, stromal cells, extracellular matrix, endothelial cells, and infiltrating leukocytes. TAM is frequently found to constitute a major part of the leukocyte infiltrates present in the tumor microenvironment (reviewed in [1]). Extensive infiltration of TAM has been shown to be positively associated with poor prognosis in breast cancer (reviewed in [15]) and high numbers of TAMs correlate with increased tumor angiogenesis and involvement of local lymph nodes in breast cancer patients $[16,17]$. Emerging evidence indicates that, in the tumor microenvironment, tumor cells affect the differentiation and functional orientation of TAM. In turn, TAMs function as a key regulator in the tumor microenvironment and evidence several characteristics of M2-Mps: the suppression of inflammatory responses and adaptive immunity, the repair and modeling of wounded and damaged tissues, and the promotion of angiogenesis (reviewed in [18]). However, there is evidence showing that the phenotype of TAMs is pliable and varies among tumor types, stages of tumor development, and the location of TAMs in the tumor microenvironment (that is, the response of TAMs to local signals) (reviewed in [19]). Understanding the roles of the specific phenotype of macrophages on tumor promotion and tumor progression is therefore important. In the present study, we demonstrated that the co-injection of M2-M $\mathrm{Ms}$ with mammary carcinoma cells into the mammary fat pad of syngeneic Balb/C mice increased solid tumor growth and lung metastasis.

In the present study, the injected green M2-M $\mathrm{M}$ s were confined to the center of the tumor tissues, whereas the $\mathrm{CD} 45^{+}$leukocytes were detected diffusely throughout the tumor tissues and increased in animals co-injected with M2-M $\varphi$ s as compared with those in mice injected with 4T1 cells alone (Figure 3A). Our results indicate that the injected macrophages were not proliferating in the tumors. Indeed, in a preliminary experiment, when M2-M $\varphi$ s were injected alone, we were unable to detect any solid mass 3 weeks after the injection when the mice were sacrificed. These results demonstrate that the crosstalk between tumor cells and M2-M the production of chemoattractants for the infiltration of circulating leukocytes. Additionally, the media conditioned by co-culture of $4 \mathrm{~T} 1$ and $\mathrm{M} 2-\mathrm{M} \varphi \mathrm{s}$ profoundly 


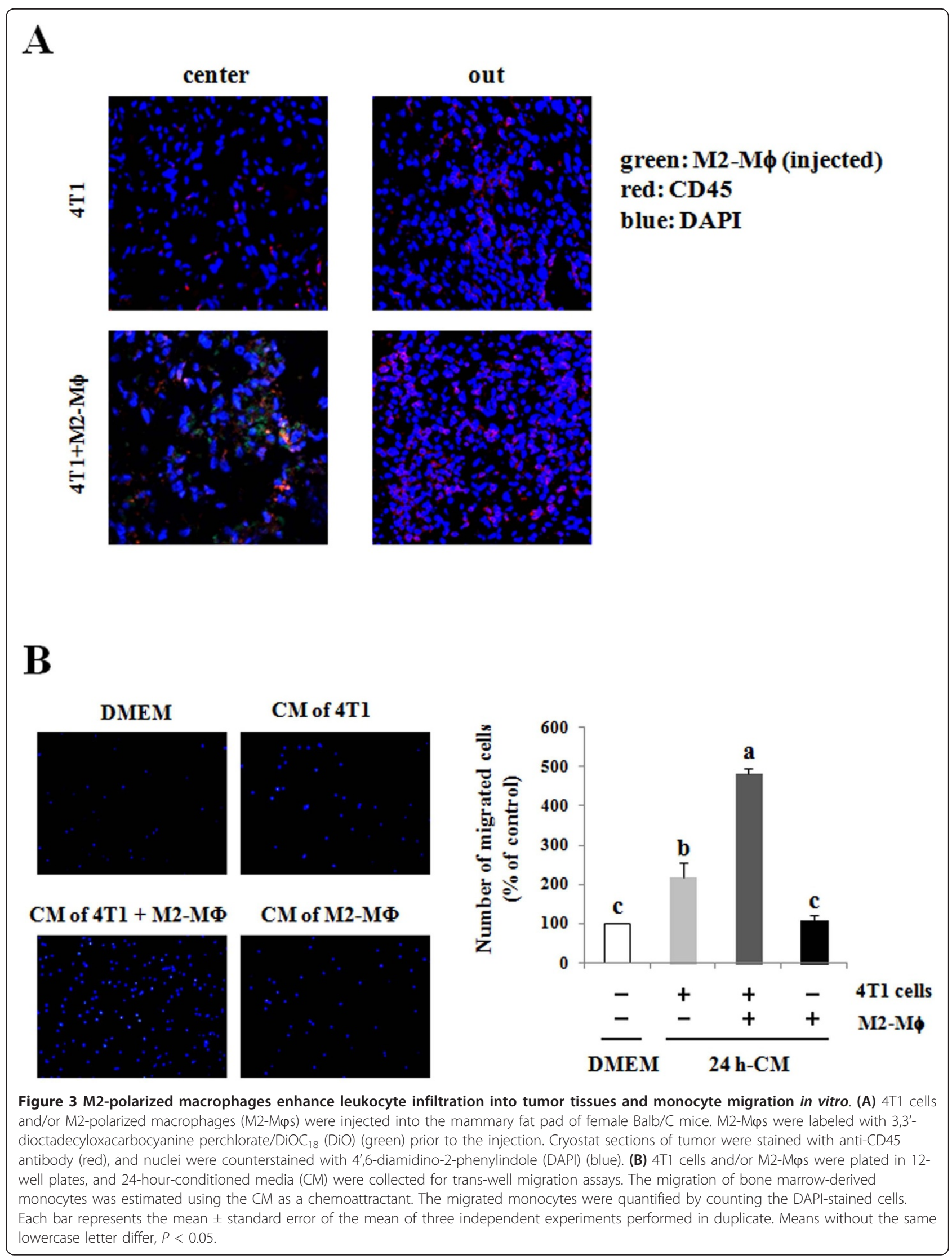


A

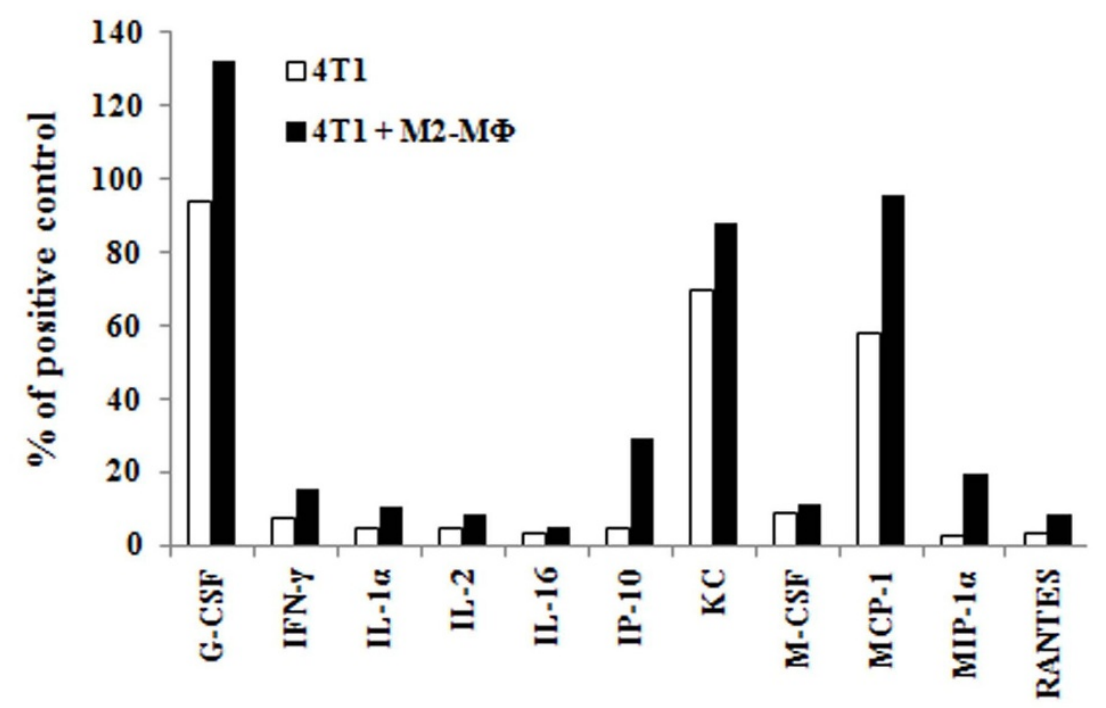

B

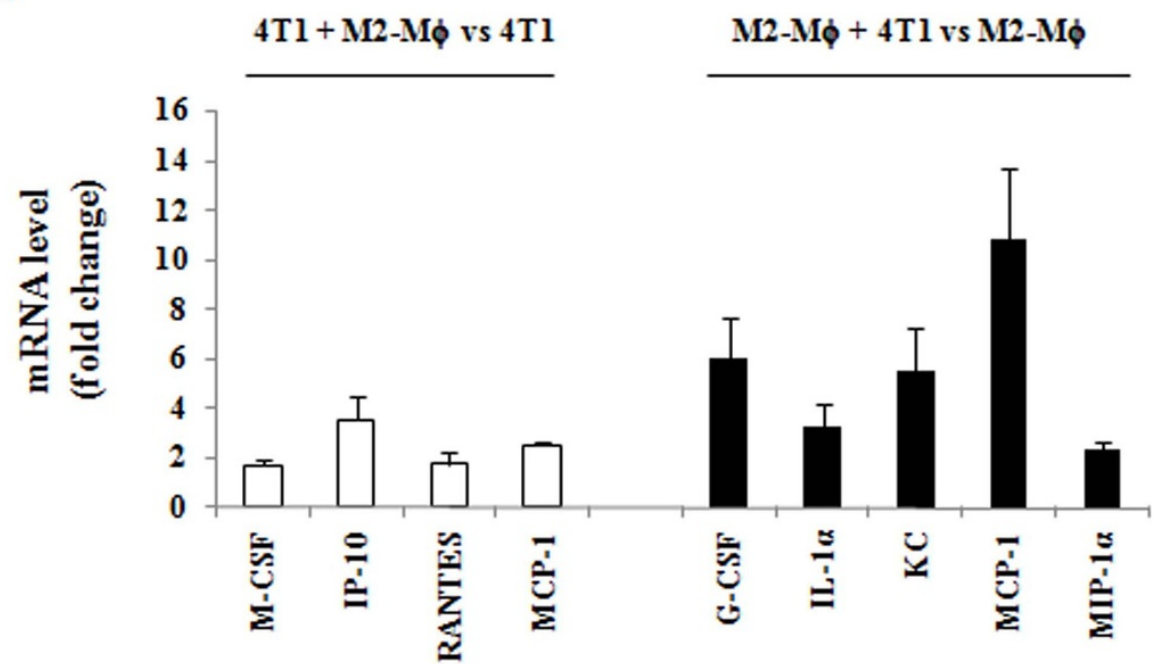

Figure 4 Crosstalk between 4T1 cells and M2-polarized macrophages stimulates cytokine production. (A) 4T1 cells and/or M2-polarized macrophages (M2-M 9 s) were plated in 12-well plates, and 24-hour-conditioned media (CM) were collected. Four independent experiments were conducted and the CM were pooled. The relative levels of cytokines in the pooled CM were estimated using a mouse cytokine array panel A kit. Expression levels were normalized to the levels of the positive control spots (contained within the membrane), and the positive control levels were set at 100\%. (B) 4T1 cells or M2-Mps were plated on cell culture coverslips (24 mm $\times 30 \mathrm{~mm}$ ) in eight-well plates. After 24 hours,

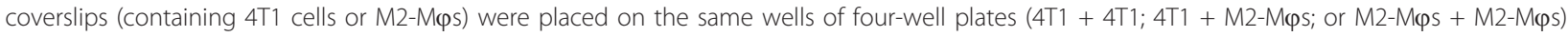
for 24 hours. Total RNA was isolated, and real-time RT-PCR was performed. Results are shown as means \pm standard error of the mean of three independent experiments performed in duplicate. Each of the control levels (4T1 cells alone or M2-Mps alone) was set to 1. G-CSF, granulocyte colony-stimulating factor; IP-10, IFN $\gamma$-induced protein-10; KC, keratinocyte chemoattractant; M-CSF, macrophage colony-stimulating factor; MCP-1, monocyte chemotactic protein-1; MIP-1 $\alpha$, macrophage inflammatory protein-1 alpha; RANTES, regulated upon activation, normal T-cell expressed and secreted.

induced the migration of bone marrow-derived monocytes (Figure $3 \mathrm{~B}$ ). The co-culture conditioned media contained higher concentrations of G-CSF, IFN $\gamma$, IL- $1 \alpha$, IL-2, IL-16, IP-10, KC, M-CSF, MCP-1, MIP-1 $\alpha$, and
RANTES as compared with media conditioned by $4 \mathrm{~T} 1$ cells alone (Figure 4A). These cytokines may have induced the infiltration of circulating monocytes into the tumor tissues and/or the stimulation of mammary 
cancer cell proliferation and metastasis in these mice. The ability of MCP-1, MIP- $1 \alpha$, and RANTES to induce the migration of human blood monocytes has been reported previously [20]. Additionally, we have previously demonstrated that M-CSF stimulated the adhesion and migration of $4 \mathrm{~T} 1$ cells under in vitro conditions [21]. The crosstalk between the two types of cells induced the mRNA expression of M-CSF, IP-10, RANTES, and MCP-1 in 4T1 cells and that of G-CSF, IL- $1 \alpha, \mathrm{KC}, \mathrm{MCP}-1$, and MIP- $1 \alpha$ in M2-M $\varphi$ s (Figure 4B). Recently, Kim and colleagues reported that the conditioned medium of $4 \mathrm{~T} 1$ cells induced the release of IL6 and TNF $\alpha$ in bone marrow-derived macrophages [22]. In the media conditioned by $4 \mathrm{~T} 1$ cells and a $4 \mathrm{~T} 1 / \mathrm{M} 2-$ $\mathrm{M} \varphi$ co-culture, IL- 6 was not detected and the co-culture induced no increase in the concentration of TNFa (data not shown). The differences between the two studies are probably attributable to differences in experimental conditions. In this study, we used a co-culture of 4T1 and IL-4-treated M2-M $\varphi$ s, whereas Kim and colleagues cultured bone marrow-derived macrophages in media conditioned by $4 \mathrm{~T} 1$ cells [22]. Future studies will be required to delineate specifically which cytokines/chemokines are responsible for the increases in the proliferation of $4 \mathrm{~T} 1$ cells and the infiltration of circulating monocytes into tumor tissues.

Immunohistochemical staining of tumor sections revealed that the proportions of Ki-67-positive cells and the expression of CDK2 and CDK4 were increased in mice co-injected with $4 \mathrm{~T} 1$ cells and $\mathrm{M} 2-\mathrm{M} \varphi$ s (Figure 1C). Moreover, the accumulation of bioluminescent signal of 4T1-luc cells was tremendously more pronounced in mice co-injected with $\mathrm{M} 2-\mathrm{M} \varphi s$ (Figure 1A,B). Furthermore, in vitro co-cultures of these two cell types revealed that $4 \mathrm{~T} 1$ cell growth was increased in the presence of M2-M $\varphi$ s (Figure 1D). The mammalian cell cycle is divided into four separate phases - the G1 phase, the $S$ phase, the $G 2$ phase, and the $M$ phase and transit through the cell cycle depends on sequential activation of CDKs. Activation of CDK4 appears to govern exit from the G0 phase and entry into an early G1 phase, and activation of CDK2 regulates transition into and passage through the S phase [23]. Overexpression of CDK2 and CDK4 was associated with breast cancer progression [24]. Taken together, the present results demonstrate that the crosstalk between 4T1 and M2$\mathrm{M} \varphi \mathrm{s}$ stimulates cell cycle progression of $4 \mathrm{~T} 1$ cells, which is probably mediated, at least in part, via the increased expression of CDK2 and CDK4. Future studies will be required to determine which cytokines are responsible for the increased growth of tumor cells.

As a tumor grows, it requires increased amounts of oxygen, resulting in hypoxia. In response to hypoxia, HIF- $1 \alpha$ accumulates and is translocated into the nucleus, where it activates the transcription of multiple genes. Additionally, HIF- $1 \alpha$ synthesis is regulated via the activation of the phosphatidylinositol 3-kinase or mitogen-activated protein kinase pathways in response to growth factors and cytokines (reviewed in [25]). HIF-1 $\alpha$ leads to the upregulation of genes involved in certain aspects of cancer progression, including angiogenesis and metastasis. VEGF-A, which is known as an important mediator of tumor angiogenesis, is one of the targets of HIF- $1 \alpha$. Recently, several studies have demonstrated that the expression of HIF- $1 \alpha$ correlates with that of VEGF-C, a lymphangiogenesis mediator $[26,27]$. We noted that the accumulation of HIF-1 $\alpha$ in the nuclei of tumor cells and the expression of VEGF-A and VEGF-C were increased in mice co-injected with $4 \mathrm{~T} 1$ cells and $\mathrm{M} 2-\mathrm{M} \varphi \mathrm{s}$ (Figure $2 \mathrm{D})$. It was reported in the $4 \mathrm{~T} 1$ syngeneic mammary cancer model that organ-specific (lung, spleen, liver, bone, and kidney) metastasis depends on the duration of tumor implant, and only lung metastasis was observed at 3 weeks after the inoculation of $4 \mathrm{~T} 1$ cells [10]. We also noted tumor nodules in the lung at 3 weeks after the $4 \mathrm{~T} 1$ cell injection. The number and volume of lung tumor nodules were increased in mice co-injected with $4 \mathrm{~T} 1$ cells and M2-M $\varphi$ s (Figure 2A to 2C). The increased expression of CD31 and lymphatic vessel endothelial hyaluronan receptor-1 in tumor tissues (Figure 2D) indicates that tumor angiogenesis and lymphangiogenesis were increased in mice co-injected with $\mathrm{M} 2-\mathrm{M} \varphi \mathrm{s}$. These increases in angiogenesis and lymphangiogenesis may, in turn, have contributed to increased solid tumor growth and lung metastasis in animals co-injected with M2-M $\varphi$ s.

\section{Conclusion}

We demonstrated that when M2-M $\varphi$ s were co-injected with mammary cancer cells into the mammary fat pads of Balb/C mice, solid tumor growth and lung metastasis were increased. The proliferation of cancer cells, the infiltration of blood leukocytes into tumor tissues, and activation of HIF- $1 \alpha$ were increased, in addition to angiogenesis and lymphangiogenesis in the tumor tissues when cancer cells were co-injected with M2-M $\varphi$ s. Our results indicate that the cross-talk between cancer cells and M2-M $\varphi s$ increased the production of chemoattractants and growth factors, which stimulated the infiltration of blood monocytes into tumor tissues and the proliferation of cancer cells, thereby increasing HIF-1 $\alpha$ activation. The activated HIF-1 $\alpha$ may also have induced the transcription of many genes involved in angiogenesis and lymphangiogenesis.

\section{Abbreviations}

CD31: platelet endothelial cell adhesion molecule-1; CDK: cyclin-dependent kinase; DiO: 3,3'-dioctadecyloxacarbocyanine perchlorate/DiOC 18 ; DMEM: Dulbecco's modified Eagle's medium; FBS: fetal bovine serum; G-CSF: 
granulocyte colony-stimulating factor; HIF-1a: hypoxia-inducible factoralpha; IFN: interferon; IL: interleukin; IP-10: IFNY-induced protein-10; KC: keratinocyte chemoattractant; M2-M $\varphi$ : M2-polarized macrophage; M-CSF: macrophage colony-stimulating factor; MCP-1: monocyte chemotactic protein-1; MIP-1a: macrophage inflammatory protein-1a; PBS: phosphatebuffered saline; PCR: polymerase chain reaction; RANTES: regulated upon activation: normal T-cell expressed and secreted; RT: reverse transcription; TAM: tumor-associated macrophage; TNF: tumor necrosis factor; VEGF: vascular endothelial growth factor.

\section{Acknowledgements}

This study was supported by the Mid-career Research Program (grant number 2012-0004903) and the SRC program (Center for Food \& Nutritional Genomics: grant number 2012-0000642) of the National Research Foundation of Korea, funded by the Ministry of Education, Science, and Technology.

\section{Author details}

'Department of Food Science and Nutrition, Hallym University, Chuncheon 200-702, Korea. ²Division of Bio-Imaging, Chuncheon Center, Korea Basic Science Institute, Chuncheon 200-701, Korea. ${ }^{3}$ Department of Biological Science, Sookmyung Women's University, Seoul 140-742, Korea.

\section{Authors' contributions}

HJC participated in the design, execution, analysis and interpretation of all results, and drafting of the manuscript. JIJ and SH participated in the design, execution, analyses, and interpretation of in vivo studies and helped to draft the manuscript. DYL and GTK participated in the design, execution, analyses, and interpretation of in vitro studies and helped to draft the manuscript. JHP participated in data interpretation and coordination of the study, and helped to draft and revise the manuscript. JHYP planned and designed this research, and drafted and revised the manuscript. All authors read and approved the final manuscript.

\section{Competing interests}

The authors declare that they have no competing interests.

Received: 31 January 2012 Revised: 14 April 2012

Accepted: 22 May 2012 Published: 22 May 2012

\section{References}

1. Allavena P, Sica A, Solinas G, Porta C, Mantovani A: The inflammatory micro-environment in tumor progression: the role of tumor-associated macrophages. Crit Rev Oncol Hematol 2008, 66:1-9.

2. Kurahara H, Shinchi H, Mataki Y, Maemura K, Noma H, Kubo F, Sakoda M, Ueno S, Natsugoe S, Takao S: Significance of M2-polarized tumorassociated macrophage in pancreatic cancer. J Surg Res 2011, 167 e211-e219.

3. Dai F, Liu L, Che G, Yu N, Pu Q, Zhang S, Ma J, Ma L, You Z: The number and microlocalization of tumor-associated immune cells are associated with patient's survival time in non-small cell lung cancer. BMC Cancer 2010, 10:220.

4. Campbell MJ, Tonlaar NY, Garwood ER, Huo D, Moore DH, Khramtsov Al, Au A, Baehner F, Chen Y, Malaka DO, Lin A, Adeyanju OO, Li S, Gong C, McGrath M, Olopade OI, Esserman LJ: Proliferating macrophages associated with high grade, hormone receptor negative breast cancer and poor clinical outcome. Breast Cancer Res Treat 2011, 128:703-711.

5. Mantovani A, Allavena P, Sica A, Balkwill F: Cancer-related inflammation. Nature 2008, 454:436-444.

6. Zhang BC, Gao J, Wang J, Rao ZG, Wang BC, Gao JF: Tumor-associated macrophages infiltration is associated with peritumoral lymphangiogenesis and poor prognosis in lung adenocarcinoma. Med Oncol 2011, 28:1447-1452.

7. Lin EY, Nguyen AV, Russell RG, Pollard JW: Colony-stimulating factor 1 promotes progression of mammary tumors to malignancy. J Exp Med 2001, 193:727-740

8. Luo Y, Zhou H, Krueger J, Kaplan C, Lee SH, Dolman C, Markowitz D, Wu W Liu C, Reisfeld RA, Xiang R: Targeting tumor-associated macrophages as a novel strategy against breast cancer. J Clin Invest 2006, 116:2132-2141.
9. Aslakson CJ, Miller FR: Selective events in the metastatic process defined by analysis of the sequential dissemination of subpopulations of a mouse mammary tumor. Cancer Res 1992, 52:1399-1405.

10. Tao K, Fang M, Alroy J, Sahagian GG: Imagable 4T1 model for the study of late stage breast cancer. BMC Cancer 2008, 8:228.

11. Kim TS, Choi HS, Ryu BY, Gang GT, Kim SU, Koo DB, Kim JM, Han JH, Park CK, Her S, Lee DS: Real-time in vivo bioluminescence imaging of lentiviral vector-mediated gene transfer in mouse testis. Theriogenology 2010, 73:129-138.

12. Weischenfeldt J, Porse B: Bone marrow-derived macrophages (BMM): isolation and applications. CSH Protoc 2008, 2008:pdb prot5080.

13. Zhang B, Wang J, Gao J, Guo Y, Chen X, Wang B, Rao Z, Chen Z: Alternatively activated RAW264.7 macrophages enhance tumor lymphangiogenesis in mouse lung adenocarcinoma. J Cell Biochem 2009, 107:134-143.

14. Cho HJ, Seon MR, Lee YM, Kim J, Kim JK, Kim SG, Park JH: 3,3'Diindolylmethane suppresses the inflammatory response to lipopolysaccharide in murine macrophages. J Nutr 2008, 138:17-23.

15. Bingle $L$, Brown NJ, Lewis CE: The role of tumour-associated macrophages in tumour progression: implications for new anticancer therapies. $J$ Pathol 2002, 196:254-265

16. Tsutsui S, Yasuda K, Suzuki K, Tahara K, Higashi H, Era S: Macrophage infiltration and its prognostic implications in breast cancer: the relationship with VEGF expression and microvessel density. Oncol Rep 2005, 14:425-431.

17. Leek RD, Landers RJ, Harris AL, Lewis CE: Necrosis correlates with high vascular density and focal macrophage infiltration in invasive carcinoma of the breast. Br J Cancer 1999, 79:991-995.

18. Sica A, Allavena P, Mantovani A: Cancer related inflammation: the macrophage connection. Cancer Lett 2008, 267:204-215.

19. Coffelt SB, Hughes R, Lewis CE: Tumor-associated macrophages: effectors of angiogenesis and tumor progression. Biochim Biophys Acta 2009, 1796:11-18.

20. Uguccioni M, D'Apuzzo M, Loetscher M, Dewald B, Baggiolini M: Actions of the chemotactic cytokines MCP-1, MCP-2, MCP-3, RANTES, MIP-1 alpha and MIP-1 beta on human monocytes. Eur J Immunol 1995, 25:64-68.

21. Kim EJ, Choi MR, Park H, Kim M, Hong JE, Lee JY, Chun HS, Lee KW, Yoon Park JH: Dietary fat increases solid tumor growth and metastasis of 4T1 murine mammary carcinoma cells and mortality in obesity-resistant BALB/c mice. Breast Cancer Res 2011, 13:R78.

22. Kim S, Takahashi H, Lin WW, Descargues P, Grivennikov S, Kim Y, Luo JL, Karin M: Carcinoma-produced factors activate myeloid cells through TLR2 to stimulate metastasis. Nature 2009, 457:102-106.

23. Foster JS, Henley DC, Ahamed S, Wimalasena J: Estrogens and cell-cycle regulation in breast cancer. Trends Endocrinol Metab 2001, 12:320-327.

24. Bonin S, Brunetti D, Benedetti E, Gorji N, Stanta G: Expression of cyclindependent kinases and CDC25a phosphatase is related with recurrences and survival in women with peri- and post-menopausal breast cancer. Virchows Arch 2006, 448:539-544.

25. Semenza GL: Targeting HIF-1 for cancer therapy. Nat Rev Cancer 2003, 3:721-732.

26. Schoppmann SF, Fenzl A, Schindl M, Bachleitner-Hofmann T, Nagy K, Gnant M, Horvat R, Jakesz R, Birner P: Hypoxia inducible factor-1a correlates with VEGF-C expression and lymphangiogenesis in breast cancer. Breast Cancer Res Treat 2006, 99:135-141.

27. Min Y, Ghose S, Boelte K, Li J, Yang L, Lin PC: C/EBP-delta regulates VEGF$C$ autocrine signaling in lymphangiogenesis and metastasis of lung cancer through HIF-1a. Oncogene 2011, 30:4901-4909.

28. Wilson SE, Mohan RR, Netto M, Perez V, Possin D, Huang J, Kwon R, Alekseev A, Rodriguez-Perez JP: RANK, RANKL, OPG, and M-CSF expression in stromal cells during corneal wound healing. Invest Ophthalmol Vis Sci 2004, 45:2201-2211.

29. Ha J, Choi HS, Lee Y, Kwon HJ, Song YW, Kim HH: CXC chemokine ligand 2 induced by receptor activator of NF-KB ligand enhances osteoclastogenesis. J Immunol 2010, 184:4717-4724.

30. Lee HS, Park JH, Kang JH, Kawada T, Yu R, Han IS: Chemokine and chemokine receptor gene expression in the mesenteric adipose tissue of KKAy mice. Cytokine 2009, 46:160-165.

31. Zhou XY, Wu P, Zhang L, Xiong W, Li YS, Feng YM, Ye DY: Effects of lipoxin $A(4)$ on lipopolysaccharide induced proliferation and reactive 
oxygen species production in RAW264.7 macrophages through modulation of G-CSF secretion. Inflamm Res 2007, 56:324-333.

32. Peinnequin A, Mouret C, Birot O, Alonso A, Mathieu J, Clarencon D, Agay D, Chancerelle Y, Multon E: Rat pro-inflammatory cytokine and cytokine related mRNA quantification by real-time polymerase chain reaction using SYBR green. BMC Immunol 2004, 5:3.

33. Takahashi Y, Li L, Kamiryo M, Asteriou T, Moustakas A, Yamashita H, Heldin P: Hyaluronan fragments induce endothelial cell differentiation in a CD44- and CXCL1/GRO1-dependent manner. J Biol Chem 2005, 280:24195-24204.

34. Oh SR, Sul OJ, Kim YY, Kim HJ, Yu R, Suh JH, Choi HS: Saturated fatty acids enhance osteoclast survival. J Lipid Res 2010, 51:892-899.

doi:10.1186/bcr3195

Cite this article as: Cho et al:: Bone marrow-derived, alternatively activated macrophages enhance solid tumor growth and lung metastasis of mammary carcinoma cells in a Balb/C mouse orthotopic model. Breast Cancer Research 2012 14:R81.

\section{Submit your next manuscript to BioMed Central} and take full advantage of:

- Convenient online submission

- Thorough peer review

- No space constraints or color figure charges

- Immediate publication on acceptance

- Inclusion in PubMed, CAS, Scopus and Google Scholar

- Research which is freely available for redistribution

Submit your manuscript at www.biomedcentral.com/submit 